\title{
Transarterial infusion chemotherapy with FOLFOX for advanced hepatocellular carcinoma: a multi-center propensity score matched analysis of real-world practice
}

\author{
Shaohua Li ${ }^{1,2 \#}$, Jie Mei ${ }^{1,2 \#}$, Qiaoxuan Wang ${ }^{2,3 \#}$, Feng Shi ${ }^{4}$, Hongyan Liu ${ }^{5}$, Ming Zhao, ${ }^{2,6}$ Lianghe Lu ${ }^{1,2}$, \\ Yihong Ling ${ }^{2,7}$, Zhixing Guo ${ }^{2,8}$, Yabing Guo ${ }^{5}$, Xiaoming Chen ${ }^{4}$, Ming Shi ${ }^{1,2}$, Wan Yee Lau', Wei Wei ${ }^{1,2}$, \\ Rongping Guo ${ }^{1,2}$
}

${ }^{1}$ Department of Liver Surgery, Sun Yat-sen University Cancer Center, Guangzhou, China; ${ }^{2}$ State Key Laboratory of Oncology in South China, Collaborative Innovation Center for Cancer Medicine, Guangzhou, China; ${ }^{3}$ Department of Radiation Oncology, Sun Yat-sen University Cancer Center, Guangzhou, China; ${ }^{4}$ Department of Interventional Radiology, Guangdong Provincial People’s Hospital, Guangdong Academy of Medical Sciences, Guangzhou, China; ${ }^{5}$ State Key Laboratory of Organ Failure Research, Guangdong Provincial Key Laboratory of Viral Hepatitis Research, Department of Infectious Diseases, Nanfang Hospital, Southern Medical University, Guangzhou, China; ${ }^{6}$ Department of Interventional Radiology, Sun Yat-sen University Cancer Center, Guangzhou, China; ${ }^{7}$ Department of Pathology, Sun Yat-sen University Cancer Center, Guangzhou, China; ${ }^{8}$ Department of Ultrasound, Sun Yat-sen University Cancer Center, Guangzhou, China; ${ }^{9}$ Faculty of Medicine, the Chinese University of Hong Kong, Shatin, New Territories, Hong Kong, China

Contributions: (I) Conception and design: R Guo, W Wei, X Chen, Y Guo, M Zhao, S Li; (II) Administrative support: None; (III) Provision of study materials or patients: None; (IV) Collection and assembly of data: S Li, J Mei, Q Wang, H Liu, F Shi, L Lu, Y Ling; (V) Data analysis and interpretation: S Li, J Mei, Q Wang, Z Guo, W Wei, R Guo; (VI) Manuscript writing: All authors; (VII) Final approval of manuscript: All authors.

\#These authors contributed equally to this work.

Correspondence to: Rongping Guo. Sun Yat-sen University Cancer Center, 651 Dongfeng East Road, Guangzhou 510000, China.

Email: guorp@sysucc.org.cn.

Background: To compare the treatment effectiveness and safety among transarterial infusion chemotherapy (TAI) with FOLFOX regimen, transarterial chemoembolization (TACE), and sorafenib in patients with BCLC stage C hepatocellular carcinoma (HCC).

Methods: The data of consecutive patients with BCLC stage C HCC treated with TAI, TACE, or sorafenib from January 2015 to December 2018 at three centers were retrospectively analyzed. Propensity-score matched (PSM) analysis was pairwise performed to reduce selection bias. Treatment effectiveness and safety were evaluated and compared using the Kaplan-Meier method, log-rank test, Cox regression models, and $\chi^{2}$ test.

Results: The median overall survival (OS) in the matched TAI cohort was significantly longer than the sorafenib cohort (19.6 vs. 7.5 months, $\mathrm{P}=0.009$ ), and the TACE cohort (estimated 27.8 vs. 6.6 months, $\mathrm{P}<0.001)$. The difference in median progression-free survival (PFS) between the matched TAI and sorafenib cohorts was not significant (5.8 vs. 2.3 months, $\mathrm{P}=0.219$ ). The median PFS in the matched TAI cohort was significantly longer than the TACE cohort (6.5 vs. 2.8 months, $\mathrm{P}<0.001)$. The objective response rate $(\mathrm{ORR})$ in the matched TAI cohort was significantly higher than the sorafenib cohort $(36.4 \%$ s. $0.0 \%, \mathrm{P}<0.001)$ and the TACE cohort ( $48.7 \%$ vs. $4.7 \%, \mathrm{P}<0.001)$. The incidences of adverse events (AEs) were similar among these three cohorts.

Conclusions: TAI with FOLFOX regimen was an effective and safe therapy that improved survival of patients with BCLC stage C HCC.

Keywords: Hepatocellular carcinoma (HCC); transarterial infusion chemotherapy; sorafenib; transarterial chemoembolization; FOLFOX

Submitted Dec 16, 2019. Accepted for publication Feb 24, 2020.

doi: 10.21037/hbsn.2020.03.14

View this article at: https://dx.doi.org/10.21037/hbsn.2020.03.14 


\section{Introduction}

Hepatocellular carcinoma (HCC) is the third leading cause of cancer death globally (1). Approximately up to over $60 \%$ of HCC patients were diagnosed at a BCLC stage C on first diagnosis $(2,3)$. The median overall survival (OS) for these patients was only 2.0-7.9 months (4-7). Currently, the recommended treatment for these patients is sorafenib $(4,5,8)$. However, low objective response rates, modest effectiveness, and high costs limit wide application of sorafenib in clinical practice $(4,5,7,9)$. In Asian countries, transarterial chemoembolization (TACE) is widely used for BCLC stage C HCC patients (10-14). TACE is not recommended for patients with distant metastasis $(8,15)$, and it should be used with caution in patients with poor liver function and in patients with main portal vein tumor thrombosis (PVTT) (16).

Recently, several studies showed transarterial infusion chemotherapy (TAI) with FOLFOX regimen alone or accompanied with sorafenib resulted in improved survival in BCLC stage C HCC patients $(17,18)$. However, the role of TAI in treatment of advanced HCC patients is still unclear when compared with the standard and more commonly used sorafenib and TACE. Herein, a multi-center propensity score-matched analysis was conducted to compare the treatment effectiveness and safety of TAI with FOLFOX regimen compared to transarterial chemoembolization (TACE), and sorafenib in patients with BCLC stage C hepatocellular carcinoma (HCC). We present the following article in accordance with the STROBE reporting checklist (available at https://dx.doi.org/10.21037/hbsn.2020.03.14).

\section{Methods}

\section{Patients}

The present study involved consecutive patients with BCLC stage C HCC treated at 3 hospitals in China (Table S1) between January 2015 to December 2018. The HCC diagnosis was made using the AASLD practice guidelines (19). The inclusion criteria were patients: (I) with Child-Pugh A or B liver function with adequate general condition; (II) who received a single treatment until radiologic response evaluation; (III) had at least one measurable lesion using the modified Response Evaluation Criteria on Solid Tumors (mRECIST) before treatment (20). Patients were excluded if they had: (I) a Child-Pugh score of C; (II) a Eastern Cooperative Oncology Group-performance score (ECOGPS) >2; (III) no measurable lesion or radiology examination before treatment, and (IV) a second malignancy. Details of patients' enrollment are shown in Figure 1.

The study was conducted in accordance with the Declaration of Helsinki (as revised in 2013). The study was approved by institutional board of Sun Yat-sen University Cancer Center (No. B2018-126-01). Informed consent was obtained from each patient for the data to be used for research.

\section{Treatment procedures}

The treatments using sorafenib, TACE, and TAI were similar to those previously reported $(10,17,18)$. In TAI group, femoral artery puncture was performed using Seldinger's technique and catheterization was performed as routine. The TAI regimen comprised of oxaliplatin, $135 \mathrm{mg} / \mathrm{m}^{2}$, from hour 0 to 3 on day 1 , leucovorin, $400 \mathrm{mg} / \mathrm{m}^{2}$, from hour 3 to 4.5 on day 1 , fluorouracil, $400 \mathrm{mg} / \mathrm{m}^{2}$, from hour 4.5 to 6.5 on day 1 , and fluorouracil, $2,400 \mathrm{mg} / \mathrm{m}^{2}$, over 46 hours from day 1 to 3 . After infusion chemotherapy was completed, the catheter and sheath were removed. TAI was performed repetitively once every 3 to 4 weeks. Any implanted port system was not applied. The regimen of TACE comprised of one to three of the following drugs: platinum, anthracycline, antibiotics, and fluorouracil, which were mixed with lipiodol.

\section{Follow-up and assessment}

Each follow-up session included a history taking, physical examination, laboratory tests, and contrast enhanced computed tomography (CT) or/and magnetic resonance imaging (MRI) examination. The initial follow-up was 1 month after TACE, 6 to 8 weeks ( 2 cycles) after TAI or 2 months after sorafenib was given. The primary endpoint was overall survival (OS), which was the time from treatment (TAI, TACE, or sorafenib) until death from any cause. The secondary endpoints included progressionfree survival (PFS), tumor response rate, post-progression survival (PPS), and treatment safety. PFS was defined as the time from treatment until disease progression or death, whichever came first. Tumor response rate included objective response rate (ORR) and disease control rate (DCR). ORR was defined as the percentage of complete response (CR) and partial response (PR) which was maintained for at least 4 weeks from the first radiological confirmation. DCR was defined as the ORR plus the percentage of patients with stable disease (SD). PPS was 


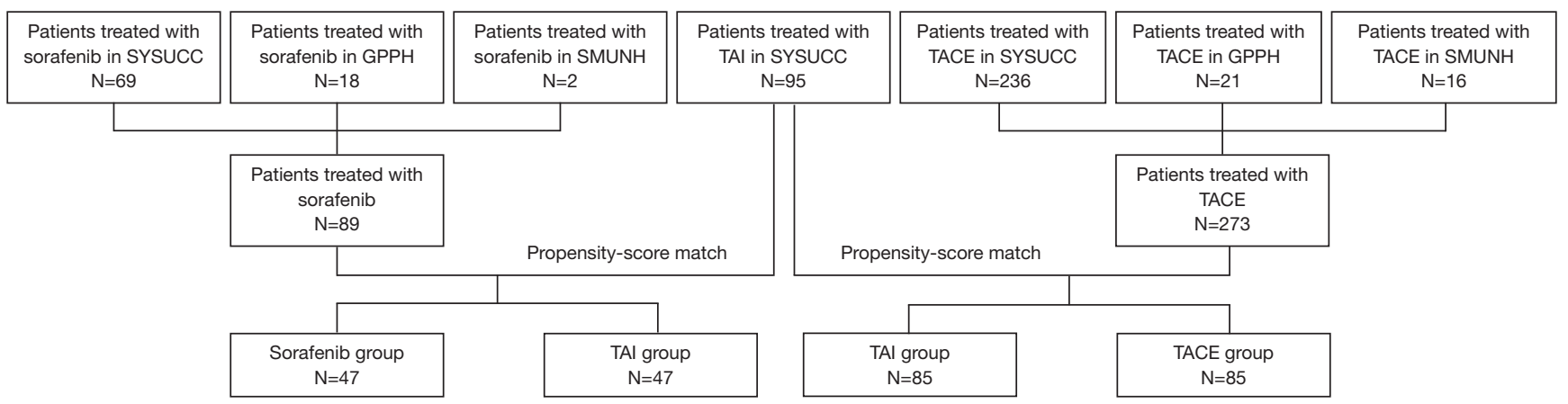

Figure 1 Flow chart of the study. SYSUCC, Sun Yat-Sen University Cancer Center; GPPH, Guangdong Provincial People's Hospital; SMUNH, Southern Medical University Nanfang Hospital.

the time from disease progression to death from any cause. Tumor response was evaluated according to the mRECIST criteria. Adverse events were graded according to the National Cancer Institute Common Terminology Criteria for Adverse Events (NCI-CTCAE) version 5.0 (21). This study was censored on May 15, 2019.

The criteria for treatment discontinuation or switch included death, refusal by patients to continue treatment, intolerable toxicities, symptomatic or radiological progression, and enough tumor-down staging to undergo a potentially radical treatment such as liver resection, liver transplantation, ablation, or stereotactic body radiotherapy (SBRT).

\section{Statistical analysis}

Categorical variables were compared using the Pearson's $\chi^{2}$ test or Fisher's exact test. Variable distributions were described using the mean \pm standard error (SE) for normally distributed values, and medium and range for non-normally distributed values. Continuous variables were compared using the Student's $t$-test for normally distributed values or the Mann-Whitney test for skewed distributed values. Survival analyses were performed using the KaplanMeier method, and differences in the survival curves were analyzed using the log-rank test. A two-tailed $\mathrm{P}<0.05$ was considered statistically significant. The propensityscore model included gender (male or female), age ( $\leq$ or $>50$ years), Child-Pugh score before treatment (A or B), serum alpha-fetoprotein (AFP) level before treatment ( $\leq$ or $>400 \mathrm{ng} / \mathrm{mL}$ ), maximum diameter of tumor $(\leq$ or $>10 \mathrm{~cm}$ ), tumor numbers (single or multiple), hemiliver involvement (unilateral or bilateral), macrovascular invasion (absent or present), and distant metastasis (absent or present). Patients who received sorafenib and TAI, and TAI and TACE were matched in the ratio of $1: 1$, with the nearest neighbor caliper of a width of 0.2. All data analyses were performed using the SPSS software, version 24.0 (SPSS Inc., Chicago, IL, USA).

\section{Results}

\section{All patients}

Between January 2015 to December 2018, the data of 457 patients (418 men and 39 women; median age, 53 years, range, 18-91 years) who were included into this study during the study period, 95, 273, and 89 underwent TAI, TACE, or sorafenib, respectively (Table S1 and Figure 1). The clinical characteristics of the entire study cohort are shown in Table 1. The ORR of patients treated in the TAI, TACE, and sorafenib groups were $44.3 \%, 7.7 \%$, and $1.3 \%$, respectively. The ORR of patients who underwent TAI was significantly higher than those who received TACE $(\mathrm{P}<0.001)$ and sorafenib $(\mathrm{P}<0.001)$. In addition, the $\mathrm{ORR}$ of patients who underwent TACE was significantly higher than those who received sorafenib $(\mathrm{P}=0.035)$. The DCR of patients treated with TAI, TACE, and sorafenib were $65.9 \%, 57.6 \%$, and $53.8 \%$, respectively. There were no significant differences among these 3 treatment groups (TAI vs. TACE, $\mathrm{P}=0.166$; TAI vs. sorafenib, $\mathrm{P}=0.108$; sorafenib vs. TACE, $\mathrm{P}=0.545)$.

The median follow-up time was 6.8 months (range, 0.2 to 52.5 months). On follow-up, 34, 202, and 50 patients treated with TAI, TACE, and sorafenib, respectively, had died; and the corresponding 55, 188, and 56 patients, respectively, had developed disease progression. The 
Table 1 Clinical characteristics of the entire cohort of patients

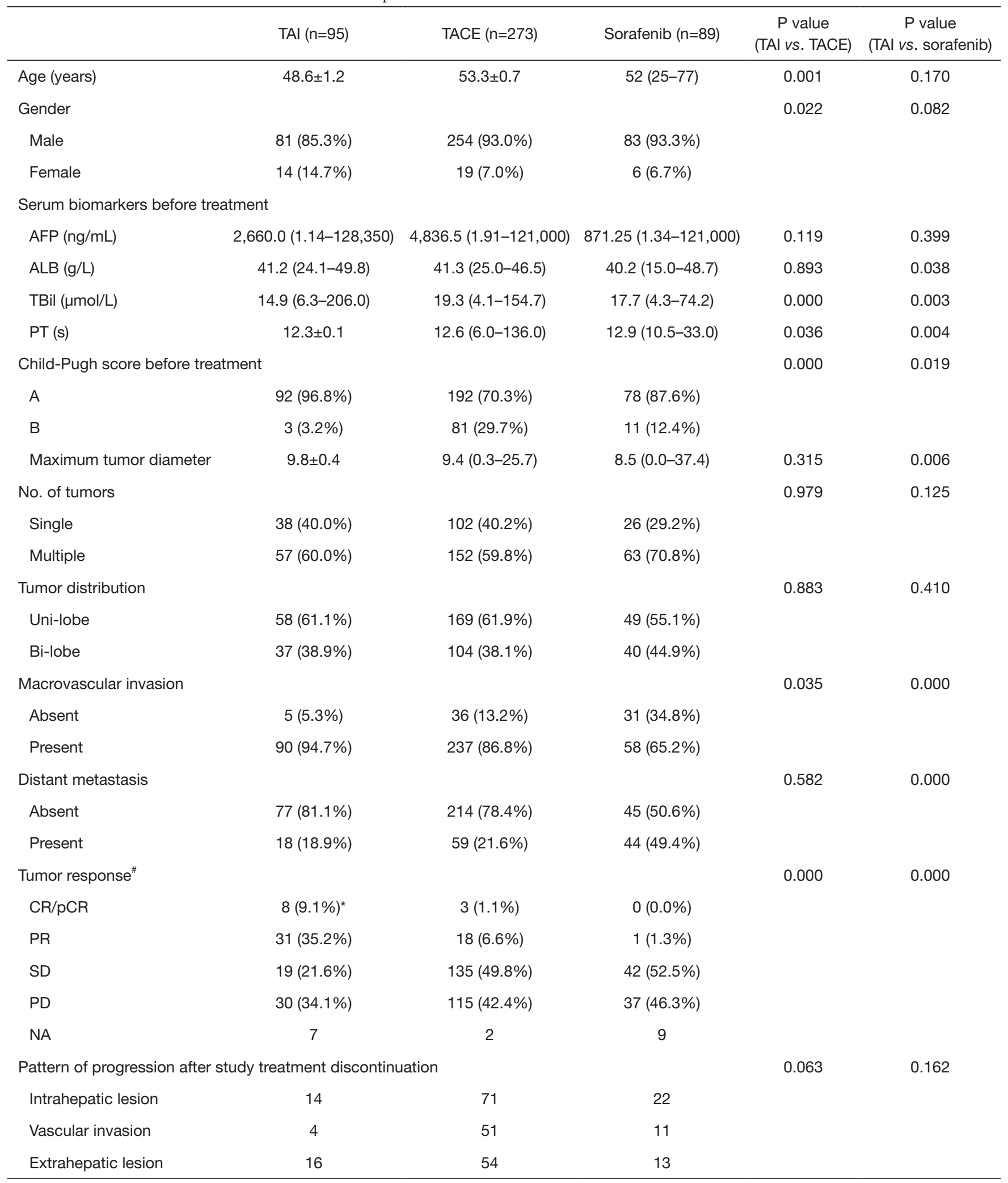

Table 1 (continued) 
Table 1 (continued)

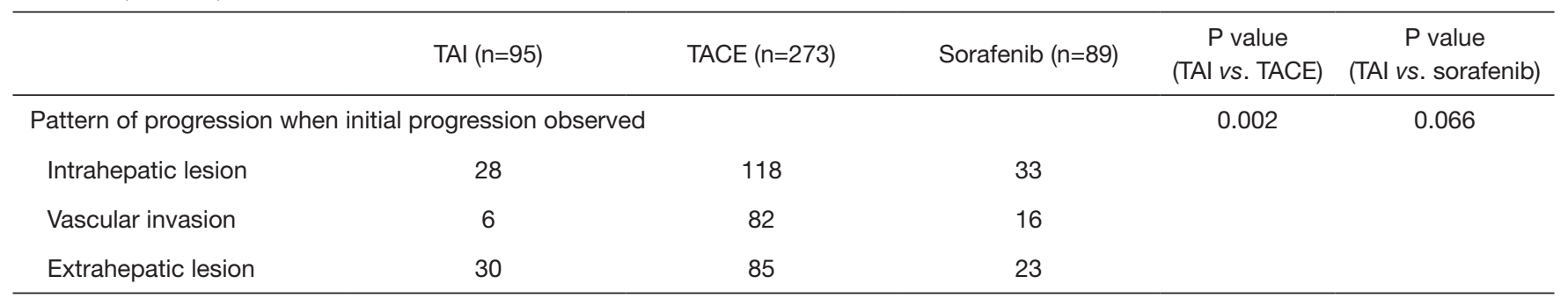

*, including 4 cases of $C R$ and 4 cases of pCR; ", TAI $(n=88)$, TACE $(n=271)$, sorafenib $(n=80)$.

A

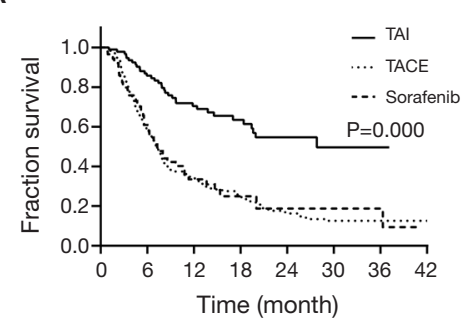

Number at risk

$\begin{array}{lllllllll}\text { TAI } & 95 & 73 & 48 & 32 & 13 & 7 & 2 & 1\end{array}$

$\begin{array}{rlccccccc}\text { TACE } & 273 & 144 & 78 & 51 & 26 & 14 & 11 & 6 \\ \text { Sorafenib } & 89 & 39 & 14 & 7 & 4 & 3 & 3 & 1\end{array}$
B

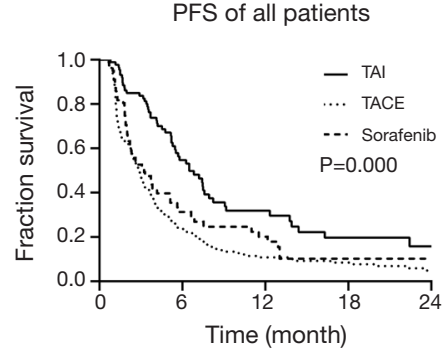

Number at risk

$\begin{array}{rlcccc}\text { TAl } & 95 & 36 & 17 & 9 & 5 \\ \text { TACE } & 273 & 38 & 17 & 12 & 6 \\ \text { Sorafenib } & 89 & 16 & 10 & 3 & 3\end{array}$

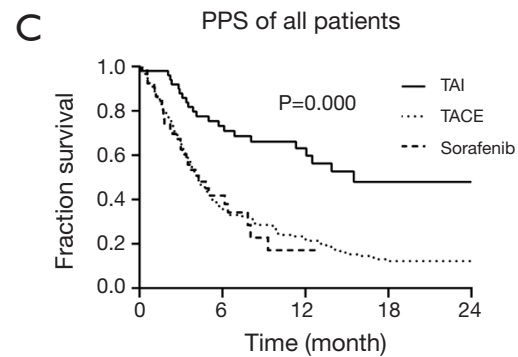

Number at risk

$\begin{array}{rlcccc}\text { TAl } & 55 & 34 & 21 & 8 & 5 \\ \text { TACE } & 188 & 58 & 35 & 18 & 12 \\ \text { Sorafenib } & 56 & 12 & 4 & 1 & 1\end{array}$

Figure 2 Survival analysis of patients from the entire cohort treated with TAI, TACE, and sorafenib. (A) OS of all patients. (B) PFS of all patients. (C) PPS of all patients.

median OS of patients treated with TAI, TACE, and sorafenib were 27.8 (estimated), 7.1 (95\% CI, 6.3-7.9), and 7.4 (95\% CI, 5.8-9.1) months, respectively. The median OS of patients who underwent TAI was significantly longer than those treated with TACE $(\mathrm{P}<0.001)$ and sorafenib $(\mathrm{P}<0.001)$. The median PFS of patients treated with TAI, TACE, and sorafenib were 6.6 (95\% CI, 5.0-8.2), 2.8 (95\% CI, 2.4-3.2), and 3.2 (95\% CI, 1.9-4.6) months, respectively. The median PFS of patients treated with TAI was significantly longer than those who were treated with TACE $(\mathrm{P}<0.001)$ and sorafenib $(\mathrm{P}=0.001)$. The median PPS of patients treated with TAI, TACE, and sorafenib were 15.5 (estimated), 4.1 (95\% CI, 3.3-4.9), and 4.3 (95\% CI, 2.8-5.7) months, respectively. The median PPS of patients treated with TAI was significantly longer than those who were treated with TACE $(\mathrm{P}<0.001)$ and sorafenib $(\mathrm{P}<0.001)$. The median OS, PFS, and PPS between patients who were treated with TACE and sorafenib were not significantly different $(\mathrm{P}=0.754,0.341$, and 0.809 , respectively) (Table S2 and Figure 2).

\section{Matched sorafenib and TAI groups}

Propensity-score matching was carried out on 47 patients in each of the sorafenib and TAI groups. The median daily dose of sorafenib was $655 \mathrm{mg}$ (296-800 $\mathrm{mg}$ ) and the median duration of medication was 69 days (20-1,101 days). The median number of cycles for TAI was 4 (1-6 cycles). The clinical characteristics of these 2 groups of patients are shown in Table 2.

The ORR of patients treated with TAI was significantly higher than those treated with sorafenib $(36.4 \%$ vs. $0.0 \%$, $\mathrm{P}<0.001$ ), while the DCR between these two groups was not significantly different (TAI vs. sorafenib group, 63.6\% vs. $47.7 \%, \mathrm{P}=0.133)$. The median $\mathrm{OS}$ of patients in the TAI group (19.6 months, 95\% CI, 12.5-26.7 months) was significantly longer than those in the sorafenib group (7.5 months, 95\% CI, 4.5-10.4 months) ( $\mathrm{P}=0.009)$. The median PFS between the sorafenib (2.3 months, 95\% CI, 1.9-2.7 months) and TAI (5.8 months, 95\% CI, 4.4-7.2 months) groups was not significantly different 
Table 2 Clinical characteristics of the matched sorafenib and TAI groups

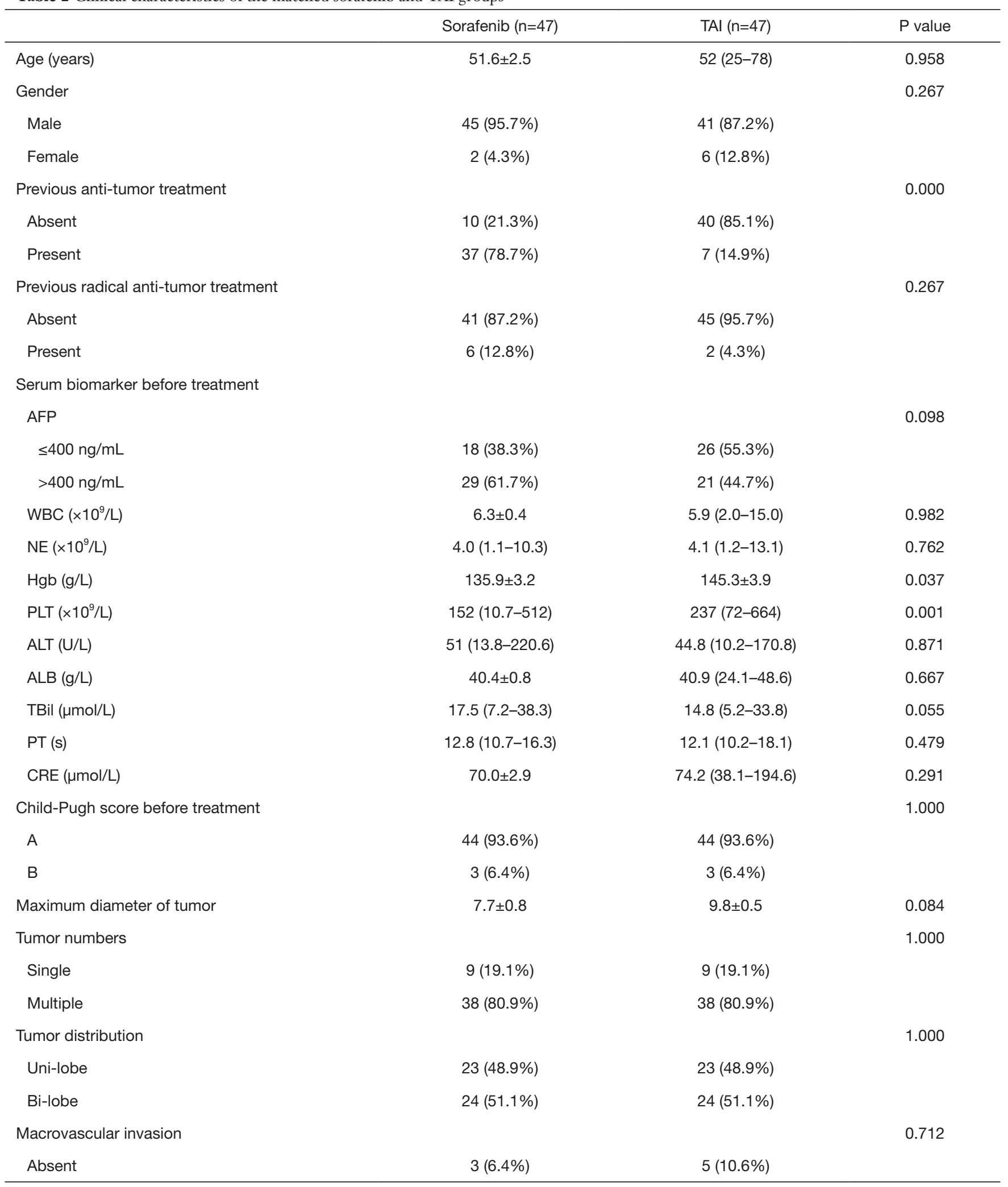

Table 2 (continued) 
Table 2 (continued)

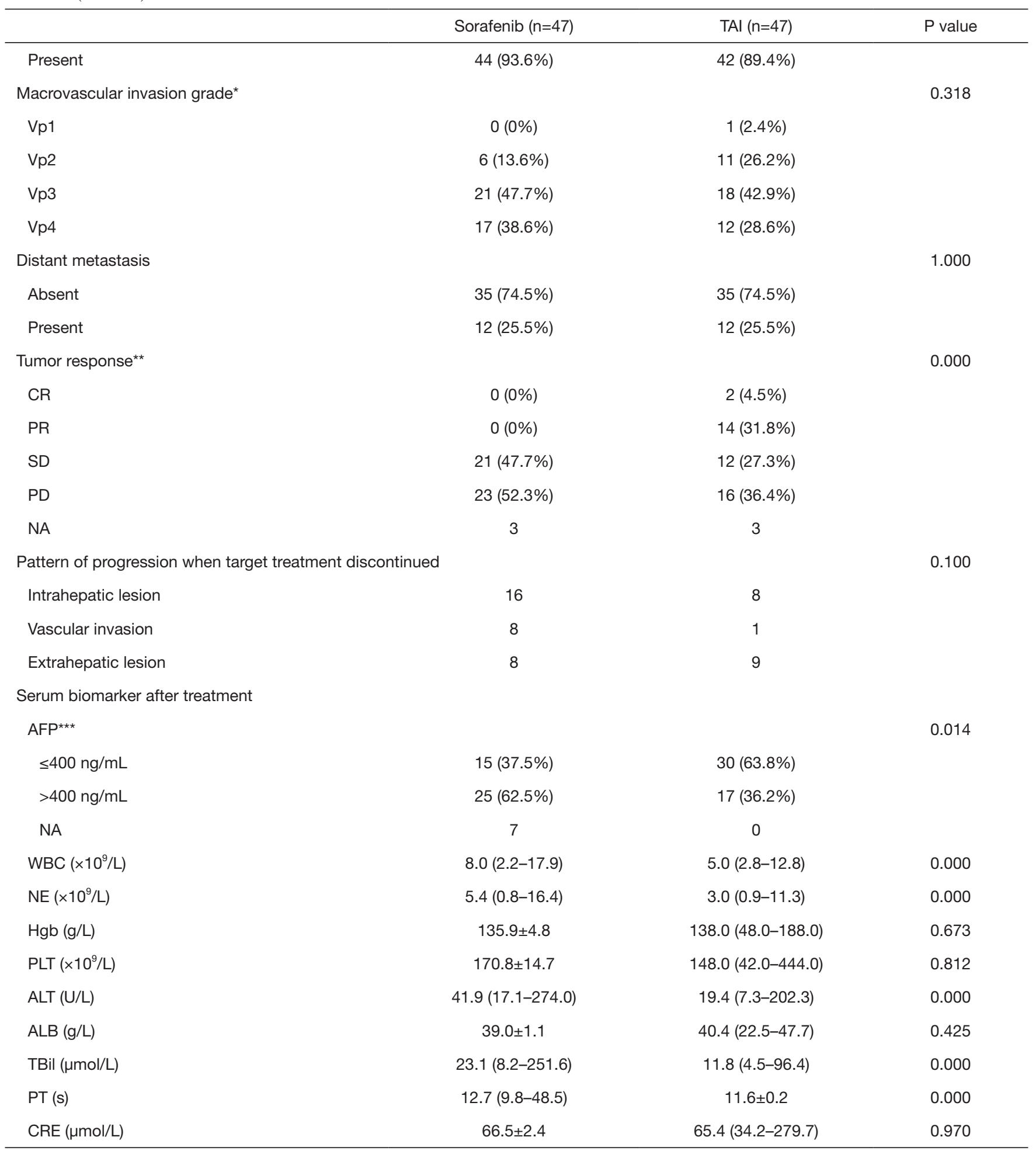

Table 2 (continued) 
Table 2 (continued)

\begin{tabular}{|c|c|c|c|}
\hline & Sorafenib $(n=47)$ & TAI $(n=47)$ & $P$ value \\
\hline A & $28(71.8 \%)$ & $44(93.6 \%)$ & \\
\hline $\mathrm{B}$ & $8(20.5 \%)$ & $3(6.4 \%)$ & \\
\hline $\mathrm{C}$ & $3(7.7 \%)$ & $0(0 \%)$ & \\
\hline Follow-up treatment & & & 0.052 \\
\hline Absent & $35(74.5 \%)$ & $26(55.3 \%)$ & \\
\hline Present & $12(25.5 \%)$ & $21(44.7 \%)$ & \\
\hline Follow-up potential radical treatment & & & 0.000 \\
\hline Follow-up surgery & & & 0.000 \\
\hline Absent & $47(100.0 \%)$ & $35(74.5 \%)$ & \\
\hline Present & $0(0.0 \%)$ & $12(25.5 \%)$ & \\
\hline Pattern of progression when initial progression observed & & & 0.032 \\
\hline Intrahepatic lesion & 22 & 18 & \\
\hline Vascular invasion & 11 & 3 & \\
\hline Extrahepatic lesion & 10 & 18 & \\
\hline
\end{tabular}

*, sorafenib $(n=44)$, TAI $(n=42) ;{ }^{* \star}$, sorafenib $(n=44)$, TAI $(n=44) ;{ }^{\star \star \star}$, sorafenib $(n=40)$, TAI $(n=47) ;{ }^{* \star \star \star}$, sorafenib $(n=39)$, TAI $(n=47)$.

A OS of matched sorafenib and TAl cohorts

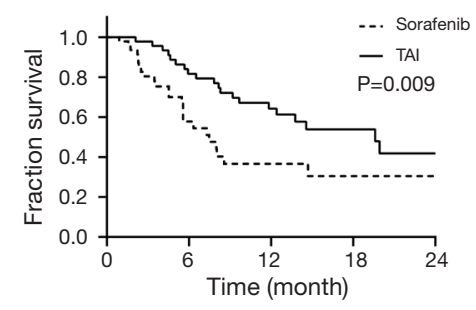

Number at risk

$\begin{array}{rrrccc}\text { Sorafenib } & 47 & 19 & 8 & 3 & 3 \\ \text { TAl } & 47 & 36 & 23 & 13 & 3\end{array}$
B
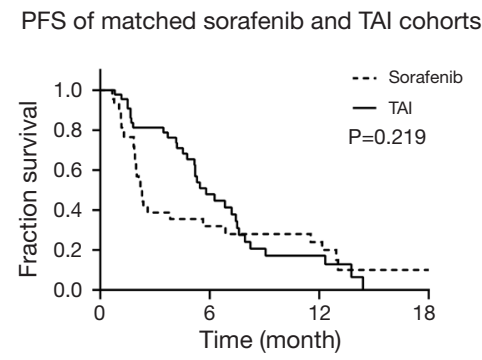

Number at risk

$\begin{array}{rr}\text { Sorafenib } & 47 \\ \text { TAl } & 47\end{array}$
C PPS of matched sorafenib and TAI cohorts

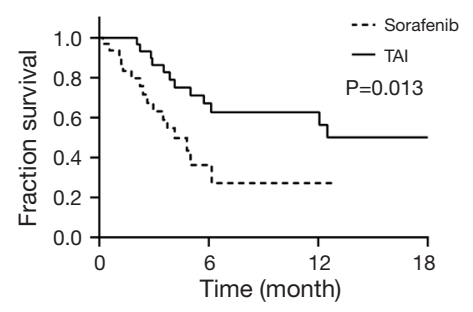

Number at risk

$\begin{array}{rcccc}\text { Sorafenib } & 33 & 5 & 3 & 1 \\ \text { TAl } & 32 & 17 & 11 & 4\end{array}$

Figure 3 Survival analysis of patients from the matched sorafenib and TAI groups. (A) OS of matched sorafenib and TAI groups. (B) PFS of matched sorafenib and TAI groups. (C) PPS of matched sorafenib and TAI groups.

$(\mathrm{P}=0.219)$. For the 33 patients in the sorafenib group and 32 patients in the TAI group included in the PPS analysis, the median PPS of patients treated with TAI (unreached) was significantly longer than those treated with sorafenib (4.1 months, 95\% CI, 2.5-5.8 months) ( $\mathrm{P}=0.013$; Figure 3).

After the pattern of disease progression was classified into three categories (intrahepatic lesion, vascular invasion, and extrahepatic lesion), PPS analysis showed that there were no significant differences between the TAI and sorafenib group in all these three patterns (Tables S3,S4).

Univariate and multivariate Cox regression analyses for OS and PFS showed grade Vp4 macrovascular invasion, 
presence of distant metastasis, AFP $>400 \mathrm{ng} / \mathrm{mL}$ after treatment, and absence of potential salvage radical treatment after tumor-downstaging were independent prognostic indicators of OS. The presence of distant metastasis, AFP $>400 \mathrm{ng} / \mathrm{mL}$ after treatment and PT $>13.5$ seconds after treatment were independent prognostic indicators of PFS in the matched sorafenib and TAI groups (Tables S5,S6).

The overall incidences of AEs were similar between the matched sorafenib and TAI groups $(\mathrm{P}=0.229$; Table S7). Leukocytopenia, neutropenia, pain, vomiting, and anorexia were more frequently found in the TAI group: $(\mathrm{P}=0.012$, $0.032,0.006,<0.001$, and 0.046 , respectively), while handfoot skin reaction (HFSR), diarrhea, hyperbilirubinemia, and prolonged prothrombin time (PT) $(\mathrm{P}=0.000,0.021$, 0.002 , and 0.019 , respectively) were more frequently found in the sorafenib group. Hypoalbuminemia, hyperbilirubinemia, anemia, pain, thrombocytopenia, and anorexia were common $(>10 \%)$ in both groups.

\section{Matched TAI and TACE groups}

After propensity-score matching, there were 85 patients in each of the TAI and TACE groups. The median number of cycles for the TAI and TACE groups were 4 (1-6 cycles) and 1 (1-4 cycles), respectively. The clinical characteristics of the two groups are shown in Table 3.

The ORR of patients in the TAI group was significantly higher than those from the TACE group (48.7\% vs. $4.7 \%$, $\mathrm{P}<0.001)$, while the DCR between these two groups showed no significant difference (TAI vs. TACE, 65.4\% vs. $56.5 \%, \mathrm{P}=0.244)$. The median OS and PFS of the TAI group were significantly longer than the TACE groups (OS, 27.8 months, estimated, vs. 6.6 months, $95 \%$ CI, 5.3-7.9 months; PFS, 6.5 months, $95 \%$ CI, 4.6-8.4 months

Table 3 Clinical characteristics of matched TAI and TACE groups

\begin{tabular}{|c|c|c|c|}
\hline & TAI $(n=85)$ & TACE $(n=85)$ & $P$ value \\
\hline Age (yr) & $49.3 \pm 1.2$ & $49[32-74]$ & 0.546 \\
\hline Gender & & & 1.000 \\
\hline Male & $77(90.6 \%)$ & $76(89.4 \%)$ & \\
\hline Female & $8(9.4 \%)$ & $9(10.6 \%)$ & \\
\hline Previous anti-tumor treatment & & & 0.794 \\
\hline Absent & $78(91.8 \%)$ & 76 (89.4\%) & \\
\hline Present & $7(8.2 \%)$ & $9(10.6 \%)$ & \\
\hline Previous radical anti-tumor treatment & & & 0.099 \\
\hline Absent & $83(97.6 \%)$ & 77 (90.6\%) & \\
\hline Present & $2(2.4 \%)$ & $8(9.4 \%)$ & \\
\hline \multicolumn{4}{|l|}{ Serum biomarker before treatment } \\
\hline AFP & & & 0.128 \\
\hline$\leq 400 \mathrm{ng} / \mathrm{mL}$ & 29 (34.1\%) & $20(23.5 \%)$ & \\
\hline$>400 \mathrm{ng} / \mathrm{mL}$ & $56(65.9 \%)$ & $65(76.5 \%)$ & \\
\hline WBC $\left(\times 10^{9} / L\right)$ & $6.2(2.0-15.0)$ & $6.4 \pm 0.3$ & 0.993 \\
\hline $\mathrm{NE}\left(\times 10^{9} / \mathrm{L}\right)$ & $4.1(1.2-13.1)$ & $4.1 \pm 0.3$ & 0.740 \\
\hline $\mathrm{Hgb}(\mathrm{g} / \mathrm{L})$ & $148.4 \pm 2.6$ & $145.3 \pm 2.4$ & 0.376 \\
\hline $\operatorname{PLT}\left(\times 10^{9} / L\right)$ & $219.0(72.0-664.0)$ & $240.6 \pm 14.8$ & 0.926 \\
\hline ALT (U/L) & $44.6(7.7-291.4)$ & $39.9(18.4-304.9)$ & 0.270 \\
\hline ALB (g/L) & $41.1(24.1-49.8)$ & $41.2 \pm 0.6$ & 0.608 \\
\hline
\end{tabular}

Table 3 (continued) 
Table 3 (continued)

\begin{tabular}{|c|c|c|c|}
\hline & TAl $(n=85)$ & TACE $(n=85)$ & $P$ value \\
\hline PT (s) & $12.3 \pm 0.1$ & $12.8 \pm 0.3$ & 0.136 \\
\hline $\mathrm{CRE}(\mu \mathrm{mol} / \mathrm{L})$ & 73.9 (41.9-194.6) & $70.9 \pm 2.6$ & 0.510 \\
\hline Child-Pugh score before treatment & & & 1.000 \\
\hline B & $2(2.4 \%)$ & $3(3.5 \%)$ & \\
\hline Maximum diameter of tumor $(\mathrm{cm})$ & $9.5 \pm 0.4$ & $11.8(1.0-16.2)$ & 0.084 \\
\hline Tumor numbers & & & 0.536 \\
\hline Single & $34(40.0 \%)$ & $39(45.9 \%)$ & \\
\hline Uni-lobe & $54(63.5 \%)$ & $62(72.9 \%)$ & \\
\hline Bi-lobe & $31(36.5 \%)$ & $23(27.1 \%)$ & \\
\hline Macrovascular invasion & & & 0.188 \\
\hline Absent & $5(5.9 \%)$ & $11(12.9 \%)$ & \\
\hline Present & $80(94.1 \%)$ & $74(87.1 \%)$ & \\
\hline Macrovascular invasion grade ${ }^{\#}$ & & & 0.903 \\
\hline Vp1 & $3(3.8 \%)$ & $3(4.1 \%)$ & \\
\hline Vp2 & $22(27.5 \%)$ & $22(29.7 \%)$ & \\
\hline Cycles & $4[1-6]$ & $1[1-4]$ & 0.000 \\
\hline Tumor response ${ }^{\# \#}$ & & & 0.000 \\
\hline $\mathrm{CR}$ (and pCR) & $8^{*}(10.3 \%)$ & $1(1.2 \%)$ & \\
\hline PR & $30(38.5 \%)$ & $3(3.5 \%)$ & \\
\hline SD & $13(16.7 \%)$ & $44(51.8 \%)$ & \\
\hline PD & $27(34.6 \%)$ & $37(43.5 \%)$ & \\
\hline NA & 7 & 0 & \\
\hline Pattern of progression when target treatment discontinued & & & 0.135 \\
\hline Intrahepatic lesion & 12 & 21 & \\
\hline Vascular invasion & 4 & 18 & \\
\hline
\end{tabular}

Table 3 (continued) 
Table 3 (continued)

\begin{tabular}{|c|c|c|c|}
\hline & TAI $(n=85)$ & TACE $(n=85)$ & $P$ value \\
\hline \multicolumn{4}{|l|}{ Serum biomarker after treatment } \\
\hline $\mathrm{AFP}^{\# \# \#}$ & & & 0.000 \\
\hline$\leq 400 \mathrm{ng} / \mathrm{mL}$ & $44(51.8 \%)$ & $15(21.4 \%)$ & \\
\hline NA & 0 & 15 & \\
\hline WBC $\left(\times 10^{9} / \mathrm{L}\right)$ & $4.9(2.3-12.8)$ & $6.7(3.5-16.9)$ & 0.000 \\
\hline $\mathrm{NE}\left(\times 10^{9} / \mathrm{L}\right)$ & $3.0(0.4-11.3)$ & $3.9(2.1-16.1)$ & 0.000 \\
\hline $\mathrm{Hgb}(\mathrm{g} / \mathrm{L})$ & $138.5(48.0-188.0)$ & $129.9 \pm 3.1$ & 0.156 \\
\hline ALB (g/L) & $40.0(22.5-47.1)$ & $38.3 \pm 0.7$ & 0.360 \\
\hline TBil $(\mu \mathrm{mol} / \mathrm{L})$ & $12.5(5.2-96.4)$ & $15.4(3.9-155.6)$ & 0.004 \\
\hline $\mathrm{PT}(\mathrm{s})$ & $11.8 \pm 0.1$ & $12.6(11.3-17.3)$ & 0.000 \\
\hline CRE $(\mu \mathrm{mol} / \mathrm{L})$ & 66.2 (34.2-279.7) & 65.6 (42.1-130.9) & 0.597 \\
\hline Child-Pugh score after treatment ${ }^{\# \# \#}$ & & & 0.168 \\
\hline A & $80(95.2 \%)$ & $48(87.3 \%)$ & \\
\hline $\mathrm{B}$ & $4(4.8 \%)$ & $7(23.7 \%)$ & \\
\hline NA & 1 & 30 & \\
\hline Present & $36(42.4 \%)$ & $16(18.8 \%)$ & \\
\hline Follow-up surgery & & & 0.000 \\
\hline Absent & $56(65.9 \%)$ & $82(96.5 \%)$ & \\
\hline Present & $29(34.1 \%)$ & $3(3.5 \%)$ & \\
\hline Pattern of progression when initial progression observed & & & 0.045 \\
\hline Intrahepatic lesion & 24 & 33 & \\
\hline Vascular invasion & 6 & 23 & \\
\hline Extrahepatic lesion & 27 & 29 & \\
\hline
\end{tabular}

*, including 4 cases of Pcr; ${ }^{\#}$, TAI $(n=80)$, TACE $(n=74) ;{ }^{\# \#, ~ T A I ~}(n=78)$, TACE $(n=85) ;{ }^{\# \#}$, TAI $(n=85)$, TACE $(n=70) ;{ }^{\# \# \#, ~ T A I ~}(n=84)$, TACE $(n=55)$. 

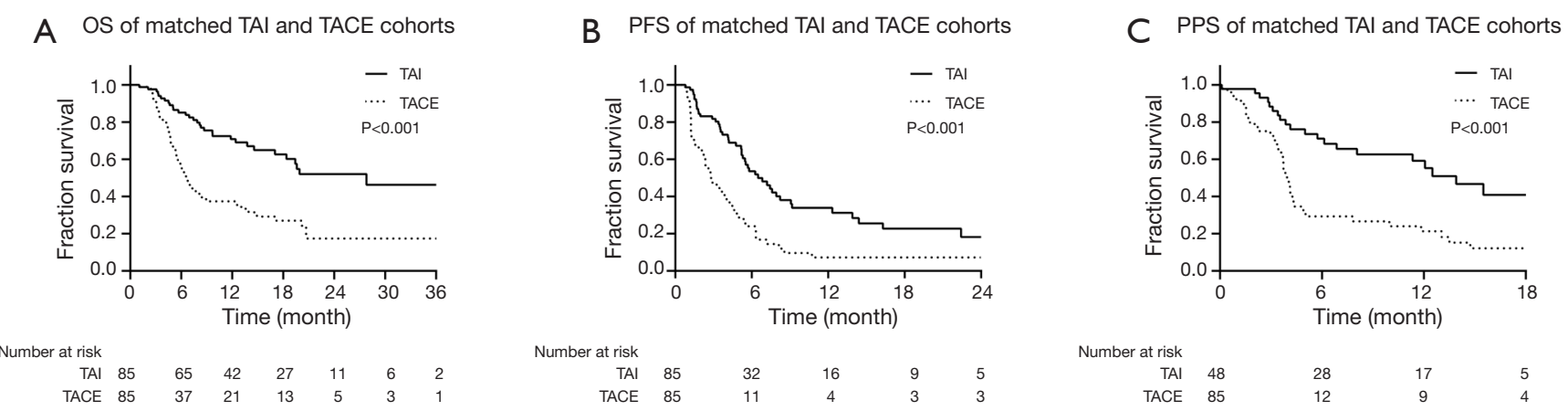

Figure 4 Survival analysis of the matched TAI and TACE groups. (A) OS of matched TAI and TACE groups. (B) PFS of matched TAI and TACE groups. (C) PPS of matched TAI and TACE groups.

vs. 2.8 months, 95\% CI, 2.0-3.6 months; both $\mathrm{P}<0.001)$. On PPS analysis for the 48 patients in the TAI group and 55 patients in the TACE group, the median PPS of the TAI group (13.9 months, 95\% CI, 9.7-18.1 months) was significantly longer than the TACE group (4.0 months, 95\% CI, 3.6-4.3 months; $\mathrm{P}<0.001$; Figure 4). Furthermore, analysis of the progression patterns showed the median PPS of the TAI group was significantly longer than the TACE group in patients having intrahepatic lesions and macrovascular invasion progression (Tables S8,S9).

Univariate and multivariate analyses for OS and PFS showed that CRE > $100 \mu \mathrm{mol} / \mathrm{L}$ before treatment, CRE $>100 \mu \mathrm{mol} / \mathrm{L}$ after treatment, and absence of potentially curative treatment after tumor-downstage were independent prognostic indicators of OS; and AFP $>400 \mathrm{ng} / \mathrm{mL}$ before treatment, AFP $>400 \mathrm{ng} / \mathrm{mL}$ after treatment, AFP level not decrease after treatment, PT $>13.5$ seconds after treatment, and absence of potentially curative treatment after tumordownstage were independent prognostic indicators of PFS in the matched TAI and TACE groups (Tables S10,S11).

The treatment-related AEs are shown in Table S12. The overall incidences of AEs were similar between the matched TAI and TACE groups $(\mathrm{P}=0.280)$. The following AEs were more frequently found in the TAI group: leukocytopenia, neutropenia, and dysuria $(\mathrm{P}=0.001,<0.001,0.006$, and 0.029 , respectively). The following AEs were more frequently found in the TACE group: elevated ALT level and fever $(\mathrm{P}=0.002$ and $<0.001$, respectively). Hypoalbuminemia, pain, vomiting, hyperbilirubinemia, anemia, thrombocytopenia, prolonged PT and anorexia were common (>10\%) in the two groups.

\section{Discussion}

Sorafenib and TACE have been recommended as the standard and the most commonly performed treatments for BCLC stage C HCC patients (4,5,8,10-12). However, limitations hampered their routine use in real-world clinical practice and an effective alternative therapy is urgently required.

The effectiveness of TAI to treat advanced HCC patients who were unable to receive TACE and percutaneous ethanol injection (PEI) was reported in 1995 by Toyoda et al. using the regimen containing cisplatin and fluorouracil. These authors reported a 1 -year OS rate of $61.1 \%$ (22). Thereafter, TAI using cisplatin, fluorouracil and with or without interferon became accepted as a common therapy to treat advanced HCC patients in Japan and Korea. However, the median overall survival was still unsatisfactory which was reported to vary between 5.3-14.0 months (23-26). The FOLFOX regimen delivered by systemic and hepatic arterial routes $(27,28)$ is commonly used in patients with colorectal cancer with liver metastasis. Several studies showed the FOLFOX regimen is also effective in patients with advanced HCC $(17,18,29)$.

For the entire cohort in the present study, the median OS, PFS and PPS, as well as the ORR and CR rate for the patients treated with TAI (44.3\% and $9.1 \%$, respectively) were significantly longer than those treated with TACE and sorafenib. In addition, 4 patients in the TAI group achieved complete pathological CR (PCR). However, the baseline clinical characteristics among the groups were unbalanced. The proportion of patients with multiple lesions and distant metastasis were highest in the sorafenib group, and the liver function of patients treated with TACE was the worst according to the Child-Pugh scoring. A propensity-score match analysis was therefore performed to reduce biases (30). The baseline tumor and prognosis related factors before treatment became comparable in the matched groups after the matching. 
One of the most important findings of the present study was that the median OS of the matched TAI group was significantly longer than the sorafenib (19.6 vs. 7.5 months, $\mathrm{P}=0.009)$ and TACE (27.8 vs. 6.6 months, $\mathrm{P}<0.001)$ groups. This result suggested that patients treated with TAI using the FOLFOX regimen was the best treatment for BCLC stage C HCC patients. The median PFS of the matched TAI group was significantly longer than the TACE group (6.5 vs. 2.8 months, $\mathrm{P}<0.001$ ), suggesting that TAI using the FOLFOX regimen resulted in better local lesion control than TACE. On the other hand, as the median PFS between the matched TAI and sorafenib groups (5.8 vs. 2.3 months, $\mathrm{P}=0.219$ ) were not significantly different, further suggesting that for advanced HCC patients, a combination of local and systemic treatments may be required to control both intra- and extra-hepatic lesions. Also, the median PPS of the matched TAI was significantly longer, which could be attributed to the smaller damage for liver function TAI made. Even if after the tumor progression, there were still more available alternative treatments, which could bring better survival.

Tumor response after TAI treatment was satisfactory in this study. The ORR was about $40 \%$ in the matched TAI group. There was even a CR rate of around $5-10 \%$ in the two matched TAI groups. A high tumor response rate brings a higher chance for potentially radical treatments, such as liver resection, transplantation and local ablative therapy with a possibility of long-term survival for advanced HCC patients (31-33). As our multidisciplinary treatment (MDT) group is led by surgeons, there is an increased awareness of the group to grasp the narrow time window for patients who could be downstaged. This explains why the proportion of patients who underwent surgery in this study was higher, and the median OS was longer than the previously reported studies on TAI using the FOLFOX regimen $(17,18)$. Several factors were identified as independent prognostic indicators of OS and PFS in both matched groups. It should be noted that AFP $>400 \mathrm{ng} / \mathrm{mL}$, PT $>13.5$ seconds, and absence of potentially curative treatment after tumor-downstage were important factors in both matched groups, suggesting that these factors were highly correlated with prognosis in advanced HCC patients. The results also showed that in the real world, for carefully selected patients with good liver function and light tumor burden, potentially curative treatment such as surgical resection could bring significant benefits for survival and disease control.

Safety analysis showed that TAI was safe for advanced HCC patients with an AEs incidence which was comparable to that of the treatments using sorafenib and TACE $(\mathrm{P}=0.229$ and 0.280 , respectively). Specifically, myelosuppression and gastrointestinal symptoms were more frequent and severe in patients treated with TAI. However, these AEs were reversible and easily manageable in clinical practice. The real concern was damage to liver function which happened slightly more often in patients treated with TAI than with other treatments. It has been shown that decompensated liver function was more important than distant metastasis in affecting long-term prognosis in patients with advanced HCC (34). Patients with good liver function have good compliance to treatment, good quality of life, and a better chance to receive alternative and effective treatments even with disease progression, thus contributing to the better survival.

The difference between the present study and the previously reported studies is on a technical point. While in our study arterial catheterization was performed in every cycle rather than using an implanted port system (24-26,35). Our approach has the benefits of better targeting tumorfeeding arteries for infusion chemotherapy, which can also be adjusted based on changes in the blood supply to tumors, thus allowing a higher local drug concentration, better pertinence, and flexibility. A treatment interval of 3-4 weeks was implemented in this study, which provided continuity of anti-tumor effects by cytotoxic agents and an enough time interval for the patients to recover liver and body functions. Also, Oxaliplatin kills cancer cells and induces immunogenic tumor cell death in a different way from cisplatin $(36,37)$.

There are limitations of this study. First, this is a retrospective study with its inherent biases despite the use of propensity-score match analysis. Second, the short follow-up might affect firm final conclusions that can be drawn from the results of this study (38). More prospective randomized controlled clinical trials on TAI, with or without other combination therapies are needed to further define the role that TAI actually plays in the treatment of patients with BCLC stage $\mathrm{C}$ hepatocellular carcinoma.

\section{Conclusions}

TAI using the FOLFOX regimen was an effective and safe treatment for BCLC stage C HCC patients. However, the results need to be validated in future prospective randomized controlled clinical trials.

\section{Acknowledgments}

Funding: This study was supported by the National 
Natural Science Foundation of China (No. 81871985); Natural Science Foundation of Guangdong Province (No. 2018A0303130098 and No. 2017A030310203); Science and Technology Planning Project of Guangdong Province (No. 2017A020215112); Medical Scientific Research Foundation of Guangdong Province (No. A2017477); Science and Technology Planning Project of Guangzhou (No. 201903010017 and No. 201904010479); Clinical Trials Project (5010 Project) of Sun Yat-sen University (No. 5010-2017009); and Clinical Trials Project (308 Project) of Sun Yat-sen University Cancer Center (No. 308-2015-014).

\section{Footnote}

Reporting Checklist: The authors have completed the STROBE reporting checklist. Available at https://dx.doi. org/10.21037/hbsn.2020.03.14

Data Sharing Statement: Available at https://dx.doi. org/10.21037/hbsn.2020.03.14

Conflicts of Interest: All authors have completed the ICMJE uniform disclosure form (available at https://dx.doi. org/10.21037/hbsn.2020.03.14). Dr. WYL serves as the unpaid editorial board members of Hepatobiliary Surgery and Nutrition. The other authors have no conflicts of interest to declare.

Ethical Statement: The authors are accountable for all aspects of the work in ensuring that questions related to the accuracy or integrity of any part of the work are appropriately investigated and resolved. The study was conducted in accordance with the Declaration of Helsinki (as revised in 2013). The study was approved by institutional board of Sun Yat-sen University Cancer Center (No. B2018-126-01).

Open Access Statement: This is an Open Access article distributed in accordance with the Creative Commons Attribution-NonCommercial-NoDerivs 4.0 International License (CC BY-NC-ND 4.0), which permits the noncommercial replication and distribution of the article with the strict proviso that no changes or edits are made and the original work is properly cited (including links to both the formal publication through the relevant DOI and the license). See: https://creativecommons.org/licenses/by-nc-nd/4.0/.

\section{References}

1. Ferlay J, Soerjomataram I, Dikshit R, et al. Cancer incidence and mortality worldwide: sources, methods and major patterns in GLOBOCAN 2012. Int J Cancer 2015;136:E359-86.

2. Poon RT, Fan ST, Lo CM, et al. Intrahepatic recurrence after curative resection of hepatocellular carcinoma: longterm results of treatment and prognostic factors. Ann Surg 1999;229:216-22.

3. Liver Cancer Study Group of Japan. Primary liver cancer in Japan. Clinicopathologic features and results of surgical treatment. Ann Surg 1990;211:277-87.

4. Cheng AL, Kang YK, Chen Z, et al. Efficacy and safety of sorafenib in patients in the Asia-Pacific region with advanced hepatocellular carcinoma: a phase III randomised, doubleblind, placebo-controlled trial. Lancet Oncol 2009;10:25-34.

5. Llovet JM, Ricci S, Mazzaferro V, et al. Sorafenib in advanced hepatocellular carcinoma. N Engl J Med 2008;359:378-90.

6. Kim BH, Lim YS, Kim EY, et al. Temporal improvement in survival of patients with hepatocellular carcinoma in a hepatitis B virus-endemic population. J Gastroenterol Hepatol 2018;33:475-83.

7. Bruix J, Cheng AL, Meinhardt G, et al. Prognostic factors and predictors of sorafenib benefit in patients with hepatocellular carcinoma: Analysis of two phase III studies. J Hepatol 2017;67:999-1008.

8. Bruix J, Sherman M; American Association for the Study of Liver Diseases. Management of hepatocellular carcinoma: an update. Hepatology 2011;53:1020-2.

9. Li L, Gou CY, Li JY, et al. Cancer of the Liver Italian Program score helps identify potential candidates for transarterial chemoembolization in patients with Barcelona Clinic Liver Cancer stage C. Hepatobiliary Pancreat Dis Int 2016;15:152-7.

10. Luo J, Guo RP, Lai EC, et al. Transarterial chemoembolization for unresectable hepatocellular carcinoma with portal vein tumor thrombosis: a prospective comparative study. Ann Surg Oncol 2011;18:413-20.

11. Kim KM, Kim JH, Park IS, et al. Reappraisal of repeated transarterial chemoembolization in the treatment of hepatocellular carcinoma with portal vein invasion. J Gastroenterol Hepatol 2009;24:806-14.

12. Yoo DJ, Kim KM, Jin YJ, et al. Clinical outcome of 251 patients with extrahepatic metastasis at initial diagnosis of hepatocellular carcinoma: does transarterial chemoembolization improve survival in these patients? J Gastroenterol Hepatol 2011;26:145-54.

13. Wei W, Jian PE, Li SH, et al. Adjuvant transcatheter arterial chemoembolization after curative resection for hepatocellular carcinoma patients with solitary tumor and microvascular invasion: a randomized clinical trial of efficacy and safety. Cancer Commun (Lond) 2018;38:61.

14. He MK, Le Y, Li QJ, et al. Hepatic artery infusion chemotherapy using $\mathrm{mFOLFOX}$ versus transarterial chemoembolization for massive unresectable hepatocellular 
carcinoma: a prospective non-randomized study. Chin J Cancer 2017;36:83.

15. Verslype C, Rosmorduc O, Rougier P, et al. Hepatocellular carcinoma: ESMO-ESDO Clinical Practice Guidelines for diagnosis, treatment and follow-up. Ann Oncol 2012;23 Suppl 7:vii41-8.

16. Raoul JL, Bruix J, Greten TF, et al. Relationship between baseline hepatic status and outcome, and effect of sorafenib on liver function: SHARP trial subanalyses. J Hepatol 2012;56:1080-8.

17. Lyu N, Kong Y, Mu L, et al. Hepatic arterial infusion of oxaliplatin plus fluorouracil/leucovorin vs. sorafenib for advanced hepatocellular carcinoma. J Hepatol 2018;69:60-9.

18. He M, Li Q, Zou R, et al. Sorafenib Plus Hepatic Arterial Infusion of Oxaliplatin, Fluorouracil, and Leucovorin vs Sorafenib Alone for Hepatocellular Carcinoma With Portal Vein Invasion: A Randomized Clinical Trial. JAMA Oncol 2019;5:953-60.

19. Bruix J, Reig M, Sherman M. Evidence-Based Diagnosis, Staging, and Treatment of Patients With Hepatocellular Carcinoma. Gastroenterology 2016;150:835-53.

20. Lencioni R, Llovet JM. Modified RECIST (mRECIST) assessment for hepatocellular carcinoma. Semin Liver Dis 2010;30:52-60.

21. Mazzaferro V, Regalia E, Doci R, et al. Liver transplantation for the treatment of small hepatocellular carcinomas in patients with cirrhosis. N Engl J Med 1996;334:693-9.

22. Toyoda H, Nakano S, Kumada T, et al. The efficacy of continuous local arterial infusion of 5-fluorouracil and cisplatin through an implanted reservoir for severe advanced hepatocellular carcinoma. Oncology 1995;52:295-9.

23. Nouso K, Miyahara K, Uchida D, et al. Effect of hepatic arterial infusion chemotherapy of 5-fluorouracil and cisplatin for advanced hepatocellular carcinoma in the Nationwide Survey of Primary Liver Cancer in Japan. Br J Cancer 2013;109:1904-7.

24. Song DS, Song MJ, Bae SH, et al. A comparative study between sorafenib and hepatic arterial infusion chemotherapy for advanced hepatocellular carcinoma with portal vein tumor thrombosis. J Gastroenterol 2015;50:445-54.

25. Saeki I, Yamasaki T, Tanabe N, et al. A new therapeutic assessment score for advanced hepatocellular carcinoma patients receiving hepatic arterial infusion chemotherapy. PLoS One 2015;10:e0126649.

26. Woo HY, Bae SH, Park JY, et al. A randomized comparative study of high-dose and low-dose hepatic arterial infusion chemotherapy for intractable, advanced hepatocellular carcinoma. Cancer Chemother Pharmacol 2010;65:373-82.

27. Allard MA, Sebagh M, Baillie G, et al. Comparison of complete pathologic response and hepatic injuries between hepatic arterial infusion and systemic administration of oxaliplatin in patients with colorectal liver metastases. Ann
Surg Oncol 2015;22:1925-32.

28. Groot Koerkamp B, Sadot E, Kemeny NE, et al.

Perioperative Hepatic Arterial Infusion Pump

Chemotherapy Is Associated With Longer Survival After Resection of Colorectal Liver Metastases: A Propensity Score Analysis. J Clin Oncol 2017;35:1938-44.

29. Qin S, Bai Y, Lim HY, et al. Randomized, multicenter, open-label study of oxaliplatin plus fluorouracil/leucovorin versus doxorubicin as palliative chemotherapy in patients with advanced hepatocellular carcinoma from Asia. J Clin Oncol 2013;31:3501-8.

30. Rosenbaum P, Rubin D. The Central Role of the Propensity Score in Observational Studies for Causal Effects. Biometrika 1983;70:41-55.

31. Peng ZW, Guo RP, Zhang YJ, et al. Hepatic resection versus transcatheter arterial chemoembolization for the treatment of hepatocellular carcinoma with portal vein tumor thrombus. Cancer 2012;118:4725-36.

32. Liang L, Chen TH, Li C, et al. A systematic review comparing outcomes of surgical resection and non-surgical treatments for patients with hepatocellular carcinoma and portal vein tumor thrombus. HPB (Oxford) 2018;20:1119-29.

33. Chen XP, Qiu FZ, Wu ZD, et al. Effects of location and extension of portal vein tumor thrombus on longterm outcomes of surgical treatment for hepatocellular carcinoma. Ann Surg Oncol 2006;13:940-6.

34. Bruix J, Raoul JL, Sherman M, et al. Efficacy and safety of sorafenib in patients with advanced hepatocellular carcinoma: subanalyses of a phase III trial. J Hepatol 2012;57:821-9.

35. Ando E, Yamashita F, Tanaka M, et al. A novel chemotherapy for advanced hepatocellular carcinoma with tumor thrombosis of the main trunk of the portal vein. Cancer 1997;79:1890-6.

36. Bruno PM, Liu Y, Park GY, et al. A subset of platinumcontaining chemotherapeutic agents kills cells by inducing ribosome biogenesis stress. Nat Med 2017;23:461-71.

37. Tesniere A, Schlemmer F, Boige V, et al. Immunogenic death of colon cancer cells treated with oxaliplatin. Oncogene 2010;29:482-91.

38. He W, Zheng Y, Zou R, et al. Long- versus short-interval follow-up after resection of hepatocellular carcinoma: a retrospective cohort study. Cancer Commun (Lond) 2018;38:26.

Cite this article as: Li S, Mei J, Wang Q, Shi F, Liu H, Zhao M, Lu L, Ling Y, Guo Z, Guo Y, Chen X, Shi M, Lau WY, Wei W, Guo R. Transarterial infusion chemotherapy with FOLFOX for advanced hepatocellular carcinoma: a multi-center propensity score matched analysis of real-world practice. HepatoBiliary Surg Nutr 2021;10(5):631-645. doi: 10.21037/hbsn.2020.03.14 